\title{
Evaluación de una maniobra nutricional tendiente a reducir los niveles de colesterol en pacientes portadores de enfermedad coronaria en el sistema público de salud chileno
}

\author{
Solange Martínez $\mathrm{O}^{\mathrm{a}}$, Yvette Zegers $\mathrm{B}^{\mathrm{a}}$, Benjamín Stockins $\mathrm{F}$, \\ Luis Bustos $\mathbf{M}^{\mathbf{b}}$, Antonio Sanhueza $\mathbf{C}^{\mathbf{b}}$, Adriana Rivera $\mathrm{S}^{\mathrm{a}}$, \\ Lidia Soto $M^{a}$, Angélica Mackay $W^{a}$, Danitza Vega $Q^{a}$, \\ Pablo Rapimán $\mathrm{D}^{\mathrm{c}}$, Rousmery Atton $\mathrm{M}^{\mathrm{c}}$, Gigliola Alberti $\mathbf{R}^{\mathrm{c}}$. \\ Evaluation of a nutritional intervention to \\ reduce cholesterol levels in patients with \\ coronary artery disease
}

Background: The mainstay of cholesterol reduction therapy is the diet. But the lack of compliance and prescription problems limit its usefulness. Aim: To compare the effectiveness of a nutritional intervention given by a nutritionist with the usual recommendations given by a physician to reduce the LDL cholesterol levels in patients with coronary artery disease, treated at the Regional public hospital in Temuco. Material and Methods: One hundred and forty patients with coronary heart disease (last acute episode at least three months before), without nutritional interventions nor cholesterol-lowering drugs, who gave informed consent, were randomized to receive either instructions by their physician or to take part in a nutritional program. The nutritional intervention consisted in five educational sessions, adapted from the NCEP and from a program of the Nutrition Department of the Catholic University of Chile. Patients randomized to the medical intervention received the standard written recommendations about diet. Lipid profile was measured before the intervention and after a three and twelve months follow up. Results: After one year the group on the nutritional program reduced $L D L$ cholesterol by $11.1 \%(p=0.03)$. There were no changes in the medical group. However, only $10 \%$ patients on the nutritional intervention group and $8 \%$ of those with medical recommendations achieved LDL cholesterol levels less than $100 \mathrm{mg} / \mathrm{dl}$. There were no changes in triglycerides, weight or body mass index during the period. Conclusions: Although this nutritional intervention proved to be more effective than usual medical instructions, most patients on secondary prevention did not achieve acceptable LDLcholesterol levels (Rev Méd Chile 2004; 132: 1457-65).

(Key Words: Coronary arteriosclerosis; Coronary disease; Lipoproteins, LDL cholesterol)

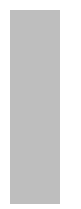

\footnotetext{
Recibido el 10 de mayo, 2004. Aceptado en versión corregida el 10 de septiembre, 2004. Departamento de Medicina Interna y CIGES, Facultad de Medicina, Universidad de La Frontera. Hospital Regional de Temuco.

Trabajo financiado por la Dirección de Investigación y Desarrollo de la Universidad de La Frontera, Proyecto DIUFRO-INI-0105.

aNutricionista, bEstadístico, 'Interno
}

Correspondencia a: Dr. Benjamín Stockins. Francisco

Salazar 01145. Temuco, Chile. Tel: (45) 325757. Fax: (45)

325777. E mail: stockins@ufro.cl 
L a enfermedad coronaria constituye una patolo_gía de curso progresivo, cuya evolución natural sólo puede ser modificada al eliminar o reducir en forma significativa sus factores de riesgo. Esta situación no sólo se da en población sana, con el objetivo de evitar su aparición, sino también en aquellos en los que ya han desarrollado la enfermedad. En este último grupo la prevención reviste aún mayor importancia, dado a que corresponde a pacientes con una mayor predisposición a desarrollar este proceso y por haber presentado ya un evento ateroesclerótico.

Hoy en día existe consenso respecto a la importancia de los niveles de lípidos sanguíneos, fijándose como meta para pacientes con enfermedad coronaria establecida, un nivel de colesterol LDL (CLDL) por debajo de los $100 \mathrm{mg} / \mathrm{dl}^{1}$. En los últimos años se han estudiado fármacos reductores de lípidos, como es el caso de las estatinas, las que han demostrado gran efectividad en prevención primaria y secundaria, tanto en la reducción de los niveles de colesterol total y CLDL, como en la disminución de nuevos eventos cardiovasculares $^{2-4}$. Desafortunadamente, su costo hace que un porcentaje muy elevado de la población no los utilice en forma rutinaria, sino sólo por algún período, en el que existe una mayor motivación ${ }^{5}$. La modificación del perfil lipídico en pacientes consultantes a instituciones públicas se hace más difícil, por no contarse con fármacos hipolipemiantes en la mayor parte de dichos establecimientos $^{6,7}$. Esto hace que la única alternativa real para muchas de estas personas, sea el modificar su perfil lipídico con una dieta rigurosa.

Los cambios nutricionales, aun cuando constituyen el pilar teórico del tratamiento para estos pacientes, no han estado exentos de cuestionamiento, por una eventual escasa efectividad y pobre adherencia en el largo plazo ${ }^{8-10}$. Sin embargo, la mayor parte de estos estudios han sido realizados en países nórdicos, con un nivel de ingreso económico claramente superior al de América Latina y con dietas que contienen mayor cantidad de grasas saturadas, lo que puede hacer más difícil la adherencia al cambio de hábitos alimentarios.

Adicionalmente, en forma frecuente, la información dietética entregada por el médico tiene un carácter vago y rutinario, no da mayores alternativas, se hace independiente del perfil lipídico completo y muchas veces se entrega a un paciente varón, el cual no tiene la responsabilidad de la preparación de los alimentos en su hogar.

Por lo anterior, el motivo de este estudio fue evaluar la efectividad de una maniobra nutricional controlada, aplicada a pacientes portadores de enfermedad coronaria en control en el Policlínico de Cardiología del Hospital Hernán Henríquez Aravena de Temuco. Dicha maniobra fue comparada con los resultados obtenidos en pacientes que sólo recibían un instructivo escrito por parte de su médico tratante durante la consulta profesional.

\section{MATERIAL y MÉTOdO}

Doscientos cuarenta y dos pacientes portadores de enfermedad coronaria (infarto del miocardio de una antigüedad de, a lo menos, tres meses, angina de pecho y personas sometidas a procesos de revascularización miocárdica) fueron invitados a participar en este ensayo clínico. El reclutamiento se estableció vía telefónica o personal, a partir de los datos de pacientes atendidos en el Policlínico de Cardiología del Centro de Diagnóstico y Tratamiento del Hospital Hernán Henríquez Aravena de la ciudad de Temuco.

Los pacientes fueron citados a dicho establecimiento, donde se les explicó las caracteństicas del estudio y se les efectuó una medición del perfil lipídico.

Se estableció como criterio de inclusión tener CLDL superior a $100 \mathrm{mg} / \mathrm{dl}$, residir en la ciudad de Temuco, no encontrarse en un programa nutricional, ni utilizar fármacos hipolipemiantes. Se excluyó a pacientes con enfermedades terminales, mentales y alcohólicos. No se hizo estudio en familiares para el diagnóstico de hipercolesterolemia familiar.

Ciento cuarenta personas $(57,8 \%)$ cumplieron con los criterios de inclusión. Del resto, 16,5\% presentaba CLDL bajo los $100 \mathrm{mg} / \mathrm{dl}, 23,9 \%$ utilizaba algún tipo de fármaco hipolipemiante y el 1,7\% no aceptó entrar al estudio.

Los pacientes incorporados fueron informados sobre la influencia de la dieta en los niveles de lípidos y su futuro riesgo cardiovascular, haciéndose hincapié en la importancia de la adherencia a las indicaciones. Firmaron consentimiento informado y posteriormente se les sometió a una encuesta que contempló: Datos personales y demográficos, presencia de factores de riesgo cardiovascular y anamnesis dietética, por recordatorio de $24 \mathrm{~h}$. Examen 
Tabla 1. C aracterísticas generales del grupo de 140 pacientes sometidos a las maniobras médica y nutricional.

\begin{tabular}{|lcrc|}
\hline & & Valor Mínimo & Valor Máximo \\
\hline Mujeres & $55,7 \%$ & - & - \\
Actividad sedentaria a liviana & $85,0 \%$ & - & - \\
No fuma & $92,8 \%$ & - & - \\
Infarto del miocardio & $68,5 \%$ & & \\
Cirugía de revascularización & $41,4 \%$ & & \\
Angioplastia coronaria & $32,8 \%$ & - & - \\
Hipertensión arterial & $81,4 \%$ & - & - \\
Diabetes Mellitus & $16,4 \%$ & - & 81 \\
IMC $\geq 25$ & $85,7 \%$ & 31 & 366 \\
Edad (años) & $62 \pm 9,8$ & 183 & 88 \\
Colesterol total (mg/dl) & $242,8 \pm 41,9$ & 113 & 880 \\
Colesterol LDL (mg/dl) & $157,1 \pm 33,6$ & 23 & 261 \\
Colesterol HDL (mg/dl) & $51,1 \pm 12,0$ & 65 & 115 \\
Triglicéridos (mg/dl) & $212,0 \pm 124,3$ & 70 & 1,8 \\
Glicemia (mg/dl) & $115,8 \pm 34,3$ & 37 & 52,9 \\
Peso & $72,7 \pm 13,4$ & 1,4 & 1,1 \\
Talla & $1,57 \pm 0,1$ & 18,6 & \\
IMC (kg/m ${ }^{2}$ Indice cintura cadera & $29,4 \pm 4,7$ & 0,7 & \\
\end{tabular}

Valores son presentados como porcentaje o promedio \pm DS.

médico y medición basal de indicadores antropométricos: peso, talla, circunferencia de cintura y cadera.

Los pacientes fueron distribuidos en forma aleatoria en dos grupos para recibir las indicaciones alimentarias, a través de una intervención nutricional controlada o médica simple. De esta manera 70 pacientes ingresaron a la maniobra nutricional y 70 a la médica. Las caracteństicas generales de los pacientes se presentan en la Tabla 1.

\section{INTERVENCIÓN}

La intervención médica, correspondió a la entrega de indicaciones alimentarias en una hoja impresa de uso habitual, aportada por una empresa farmacéutica, la cual contempla los rubros de alimentos separados de acuerdo a su grado de recomendación (uso libre, moderado y restringido).

La maniobra nutricional controlada, comprendió una intervención dietoterapéutica, enmarcada dentro de la política nacional del Ministerio de Salud. Consistió en cinco sesiones desarrolladas a partir del programa del Departamento de Nutrición de la Pontificia Universidad Católica de Chile, que incorpora las recomendaciones de la dieta tipo II del NCEP (National Cholesterol Education Program) y que fue adaptada a la realidad social de los consultantes del Hospital Regional de Temuco.

Esta dieta contempla calorías según diagnóstico nutricional (tasa metabólica basal por peso aceptable según talla y sexo, según Metropolitan Life Insurance Company) ${ }^{11}$. La distribución de macronutrientes y fibra puede observarse en la Tabla 2.

Tabla 2. Recomendaciones calóricas y nutricionales del grupo, estimadas sobre la base del peso aceptable según promedio de estatura

\begin{tabular}{|lcc|}
\hline & Masculino & Femenino \\
\hline Calorías (Kcal/día) & $1.573 \pm 158$ & $1.173 \pm 116$ \\
Hidratos de carbono & $55 \%$ VCT \\
Proteínas & $15 \%$ VCT \\
Lípidos & $30 \%$ VCT \\
Grasa poli-insaturada & $\leq 10 \%$ VCT \\
Grasa mono-insaturada & $\leq 20 \%$ VCT \\
Grasa saturada & $<7 \%$ VCT \\
Fibra (gramos/día) & $>25$ \\
Colesterol (miligramos/día) & $<200$ \\
\hline
\end{tabular}

VCT $=$ Valor Calórico Total. Estatura promedio en hombres fue de $1,64 \mathrm{~m}$ y $1,51 \mathrm{~m}$ en mujeres. 
La maniobra contempló cinco sesiones en las que se estableció una anamnesis dietética, seguida de la entrega de una pauta alimentaria y de elementos educativos, que incorporaban características en la preparación de los alimentos y sus eventuales reemplazos. Las sesiones tuvieron carácter individual en tres ocasiones y grupal en dos. Los varones, fueron acompañados por su esposa u otro familiar encargado de la preparación de los alimentos.

Los pacientes fueron citados a los tres y doce meses, con el objeto de repetir las mediciones efectuadas en forma basal. Aquellos adscritos al programa nutricional recibieron, al tercer mes, un refuerzo educativo de dos sesiones.

El perfil lipídico fue realizado en ayunas y analizado por método enzimático en Laboratorio SIRESA de Temuco, por contar éste con un control de calidad certificado por el Instituto de Salud Pública de Chile y del College of American Pathologists U.S.A. El CLDL fue medido en forma directa.

Estadística. El tamaño de la muestra fue calculado considerando que este grupo de pacientes tiene niveles de CLDL de $184+49,1 \mathrm{mg} / \mathrm{dl}^{6,7}$, asumiendo un poder de $80 \%$ y un nivel de significación de $5 \%$, para probar la hipótesis de diferencia promedio de disminución del CLDL entre el tratamiento nutricional y convencional o médico de a lo menos $10 \%$ (25 mg/dl) entre los grupos. El tamaño de muestra calculado fue de 70 en cada grupo.

Los datos fueron almacenados en una base de datos Excel. El análisis estadístico se llevó a cabo con el programa Stata versión 8.0, aplicando la prueba t-test y $\chi^{2}$ para comparar las diferencias entre las medias de los grupos, con un nivel de significancia $\alpha=0,05$.

El grupo de trabajo estuvo formado por médico, nutricionistas y alumnos de las carreras de la salud de la Facultad de Medicina de la Universidad de la Frontera.

\section{RESULTADOS}

El promedio de edad del grupo fue 62 años, destacando una alta prevalencia de malnutrición por exceso (IMC $\geq 25$ ) en $85,7 \%$ de los pacientes.
Ochenta y cinco porciento de los pacientes mantenía una actividad física sedentaria a liviana y 92,8\% no fumaba, $81,4 \%$ presentaba hipertensión arterial y 16,4\% diabetes mellitus. El promedio de CLDL fue $157,1 \pm 33,6 \mathrm{mg} / \mathrm{dl}$, muy por encima de las recomendaciones para estos pacientes (Tabla 1).

Sólo 21,2\% presentaba cifras de CLDL bajo 130 $\mathrm{mg} / \mathrm{dl}$.

La Tabla 3 muestra las características basales biodemográficas, clínicas y bioquímicas de cada grupo, sin existir diferencia significativa entre ellos.

La Tabla 4 muestra la comparación entre la ingesta real de calorías y macronutrientes observada, comparada con las recomendaciones calculadas según gasto energético basal y el peso aceptable. Se observa que ambos grupos tenían una alimentación hipercalórica, alta en hidratos de carbono, normo proteica y normo lipídica. En este último aspecto había una distribución mayor de grasas polinsaturadas en desmedro de las monoinsaturadas.

Seguimiento. De los 140 pacientes reclutados, $83 \%$ acudió a control al tercer mes (88\% en el grupo médico y $77 \%$ en el nutricional) y sólo $73,5 \%$ al año (74\% en el grupo médico y $73 \%$ en el nutricional). En relación a esta adherencia, no se observó diferencias por sexo $(55,5 \%$ en mujeres y $44,5 \%$ en varones) ni por aleatorización.

La Tabla 5 muestra los niveles de lípidos al momento inicial del estudio, y el porcentaje de cambio tras tres y doce meses de dieta. Se puede observar que hubo reducción no significativa en los niveles de colesterol total, sin diferencias en ambos grupos. El CLDL al tercer mes alcanzó una reducción de $5,7 \%(p=0,002)$ en el grupo con maniobra nutricional comparado con $0,7 \%$ en el que sólo recibió instrucción médica (NS). Al año, el grupo con intervención nutricional logró una reducción del CLDL de $11,1 \%(p=0,03)$. En el grupo con intervención médica se apreció un alza de $3,1 \%$ (NS).

Aun cuando las cifras de CHDL presentaron en el grupo nutricional un alza al tercer mes, ello no persistió en el control de los doce meses. Ninguno de los grupos logró disminuir en forma significativa las cifras de triglicéridos al año. 
Tabla 3. C aracterísticas basales biodemográficas, de indicadores antropométricos y bioquímicos de los dos grupos sometidos a maniobra nutricional o médica

\begin{tabular}{|lcc|}
\hline & Nutrición & Médico \\
\hline $\mathrm{N}$ & 70 & 70 \\
Edad (años) & $59,8 \pm 9,6$ & $64,2 \pm 9,7$ \\
Mujeres & $42(60 \%)$ & $36(51 \%)$ \\
Antecedentes mórbidos personales: & & \\
$\quad$ Diabetes Mellitus & $12(17 \%)$ & $11(16 \%)$ \\
Hipertensión arterial & $54(77 \%)$ & $60(86 \%)$ \\
Tabaco & $2(3 \%)$ & $8(11 \%)$ \\
Alcohol & $24(34 \%)$ & $33(47 \%)$ \\
Actividad física: & & \\
$\quad$ Sedentaria a liviana & $62(88 \%)$ & $57(81 \%)$ \\
Moderada a intensa & $8(12 \%)$ & $13(19 \%)$ \\
IMC $\geq 18,5 \leq 24,9$ & $8(11 \%)$ & $12(17 \%)$ \\
IMC $\geq 25 \leq 29,9$ & $30(43 \%)$ & $26(37 \%)$ \\
IMC $\geq 30$ & $32(46 \%)$ & $32(46 \%)$ \\
IMC promedio & $29,1 \pm 3,6$ & $29,7 \pm 5,7$ \\
Indice cintura cadera & $0,9 \pm 0,06$ & $0,9 \pm 0,07$ \\
Colesterol total (mg/dl) & $249,2 \pm 39,8$ & $236,4 \pm 43,3$ \\
Colesterol-LDL (mg/dl) & $161,8 \pm 31$ & $152,4 \pm 35,6$ \\
Colesterol-HDL (mg/dl) & $50,5 \pm 13,2$ & $51,6 \pm 10,9$ \\
Triglicéridos (mg/dl) & $235,1 \pm 147,1$ & $188,9 \pm 91,8$ \\
Glicemia (mg/dl) & $115,0 \pm 30,2$ & $116,7 \pm 38,2$ \\
Hb glicosilada & $8,8 \pm 0,8$ & $7,1 \pm 0,9$ \\
& & \\
\hline
\end{tabular}

Valores son presentados como número, porcentaje o promedio \pm DS.

Cabe hacer mención que sólo $10 \%$ del grupo nutricional y $8 \%$ del grupo médico, alcanzó al año la meta de un CLDL por debajo de los $100 \mathrm{mg} / \mathrm{dl}$ (Tabla 6). La Tabla 7 muestra la distribución de los pacientes de acuerdo a tres niveles de CLDL basales y el porcentaje de ellos que logró cifras menores a $100 \mathrm{mg} / \mathrm{dl}$ al año. Se aprecia que incluso en aquellos con valores cercanos a la meta, el porcentaje que logra fue bajo.

No existió una relación entre los niveles de lípidos y el IMC. Tampoco se observó una modificación del peso ni del IMC tras la administración de las maniobras en los dos grupos. Sin embargo, el contorno de cintura disminuyó en el grupo nutricional de 97,7 a 94,7 cm $(p=0,01)$.
Tabla 4. Relación porcentual entre la cobertura calórica y nutricional encontrada y la recomendada en cada grupo de intervención

\begin{tabular}{|lrc|}
\hline & Nutrición & Médico \\
\hline Calorías & $128 \%$ & $142 \%$ \\
Hidratos de carbono & $148 \%$ & $163 \%$ \\
Proteínas & $105 \%$ & $111 \%$ \\
Lípidos & $92 \%$ & $108 \%$ \\
Grasa poliinsaturada & $154 \%$ & $154 \%$ \\
Grasa monoinsaturada & $2,2 \%$ & $0,6 \%$ \\
Grasa saturada & $33 \%$ & $49 \%$ \\
\hline
\end{tabular}


Tabla 5. M odificación del perfil lipídico en los grupos sometidos a maniobra nutricional o médica al cabo de tres y doce meses, expresados como mediana y media

\begin{tabular}{|lcccc|}
\hline & \multicolumn{2}{c}{ Nutrición } & \multicolumn{2}{c|}{ Médico } \\
& Mediana $+\mathrm{RI}$ & Media $\pm \mathrm{DS}$ & Mediana $\pm \mathrm{RI}$ & Media $\pm \mathrm{DS}$ \\
\hline Colesterol total & & & & \\
Basal & $246,0 \pm 44$ & $249,2 \pm 39,8$ & $227,0 \pm 43,0$ & $236,4 \pm 43,0$ \\
Tercer mes & $237,0 \pm 48,0$ & $241,1 \pm 42,3$ & $217,0 \pm 64,0$ & $227,4 \pm 46,2$ \\
Año & $215,0 \pm 56,0$ & $231,1 \pm 66,8$ & $229,0 \pm 85,0$ & $234,9 \pm 48,0$ \\
Tercer mes v/s Basal $\Delta \%$ & $-3,6$ & $-3,2(\mathrm{p}=0,02)$ & $-4,4$ & $-3,8(\mathrm{p}=0,01)$ \\
Año v/s basal $\Delta \%$ & $-12,6$ & $-7,2(\mathrm{p}=0,2)$ & $+0,9$ & $-0,6(\mathrm{p}=0,42)$ \\
Colesterol LDL & & & & \\
Basal & $155,0 \pm 34,0$ & $161,8 \pm 31,0$ & $141,5 \pm 50,0$ & $152,4 \pm 35,6$ \\
Tercer mes & $149,0 \pm 58,0$ & $152,5 \pm 38,6$ & $145,0 \pm 57,0$ & $151,2 \pm 49,7$ \\
Año & $142,0 \pm 45,0$ & $143,9 \pm 42,4$ & $148,0 \pm 40,0$ & $147,6 \pm 37,3$ \\
Tercer mes v/s basal $\Delta \%$ & $-3,9$ & $-5,7(\mathrm{p}=0,002)$ & $+2,5$ & $-0,7(\mathrm{p}=0,78)$ \\
Año v/s basal $\Delta \%$ & $-8,4$ & $-11,1(\mathrm{p}=0,03)$ & $+4,6$ & $3,1(\mathrm{p}=0,84)$ \\
Colesterol HDL & & & & \\
Basal & $49,0 \pm 19,0$ & $50,4 \pm 13,1$ & $49,0 \pm 13,0$ & $51,6 \pm 10,8$ \\
Tercer mes & $51,0 \pm 11,0$ & $54,8 \pm 13,9$ & $45,0 \pm 11,0$ & $48,3 \pm 9,1$ \\
Año & $46,0 \pm 12,0$ & $48,6 \pm 11,2$ & $48,0 \pm 10,5$ & $48,1 \pm 9,9$ \\
Tercer mes v/s Basal $\Delta \%$ & $+4,1$ & $+8,7(\mathrm{p}=0,009)$ & $-8,2$ & $-6,3(\mathrm{p}=0,004)$ \\
Año v/s Basal $\Delta \%$ & $-6,1$ & $-3,6(\mathrm{p}=0,9)$ & $-2,0$ & $-6,8(\mathrm{p}=0,007)$ \\
Triglicéridos & & & & \\
Basal & $186,0 \pm 191,0$ & $235,1 \pm 147,1$ & $167,0 \pm 129,0$ & $188,9 \pm 91,8$ \\
Tercer mes & $176,0 \pm 181$ & $208,1 \pm 140,1$ & $154,0 \pm 112,0$ & $186,8 \pm 100,0$ \\
Año & $171,0 \pm 154,0$ & $221,8 \pm 134,5$ & $161,0 \pm 95,5$ & $195,1 \pm 91,2$ \\
Tercer mes v/s Basal $\Delta \%$ & $-5,4$ & $-11,5(\mathrm{p}=0,001)$ & $-7,8$ & $-1,1(\mathrm{p}=0,61)$ \\
Año v/s Basal $\Delta \%$ & $-8,1$ & $-5,6(\mathrm{p}=0,63)$ & $-3,6$ & $3,3(\mathrm{p}=0,28)$ \\
& & & & \\
\hline
\end{tabular}

Tabla 6. D istribución porcentual del grupo nutrición y médico en el logro de metas de CLD L al cabo de un año

\begin{tabular}{|lrrr|}
\hline & Nutrición & Médico & Valor $\mathrm{p}$ \\
\hline Colesterol LDL $<100 \mathrm{mg} / \mathrm{dl}$ & $5(10 \%)$ & $4(8 \%)$ & 0,722 \\
$100 \mathrm{mg} / \mathrm{dl} \leq \mathrm{colesterol} \mathrm{LDL}<130 \mathrm{mg} / \mathrm{dl}$ & $14(27 \%)$ & $14(28 \%)$ & 0,861 \\
Colesterol LDL $\geq 130 \mathrm{mg} / \mathrm{dl}$ & $32(63 \%)$ & $32(64 \%)$ & 0,935 \\
& $51(100 \%)$ & $50(100 \%)$ & \\
\hline
\end{tabular}

Los resultados se expresan en porcentaje con respecto al número total de cada grupo.

Tabla 7. D istribución porcentual del grupo nutrición y médico que logra CLD L menor a $100 \mathrm{mg} / \mathrm{dl}$ al cabo de un año, en relación a sus niveles basales

\begin{tabular}{|c|c|c|c|}
\hline Colesterol LDL basal & $\begin{array}{r}\text { Colester } \\
\text { al ca } \\
\text { Nutrición }\end{array}$ & $\begin{array}{l}00 \mathrm{mg} / \mathrm{dl} \\
\text { año } \\
\text { Médico }\end{array}$ & Valor $\mathrm{p}$ \\
\hline C LDL 100-129 mg/dl & $33 \%$ & $21 \%$ & NS \\
\hline C LDL 130-159 mg/dl & $9 \%$ & $11 \%$ & NS \\
\hline C LDL $\geq 160 \mathrm{mg} / \mathrm{dl}$ & $2,4 \%$ & $0 \%$ & NS \\
\hline
\end{tabular}




\section{DisCUSIÓN}

Evidencias clínicas y epidemiológica, sustentan el hecho que una dieta pobre en grasas saturadas y colesterol, disminuye el riesgo de enfermedad coronaria y muerte por esta causa ${ }^{12-14}$. Asimismo, numerosas publicaciones avalan la efectividad en la reducción de eventos coronarios al reducir niveles de lípidos con el uso de fármacos en pacientes portadores de enfermedad coronaria, así como también en prevención primaria ${ }^{2-4,15}$.

Desafortunadamente, los pacientes portadores de cardiopatía coronaria tienden a mantener sus factores de riesgo cardiovascular. En un estudio reciente, estudiamos a pacientes que habían sido sometidos a procesos de revascularización miocárdica, observando que $87 \%$ de ellos presentaba niveles de CLDL por sobre los $100 \mathrm{mg} / \mathrm{dl}$ y $66 \%$ cifras por sobre los $130 \mathrm{mg} / \mathrm{dl}$. De éstos, sólo 38\% se encontraba utilizando fármacos hipolipemiantes, dadas las dificultades económicas para adquirirlos $^{6,7}$. La prevalencia de otros factores de riesgo (tabaco, obesidad, diabetes mellitus e hipertensión arterial) fue similar a los de este grupo de pacientes. No hemos efectuado estudios de hiperlipidemia familiar, ya que no se trata de una práctica rutinaria en nuestra Unidad de Cardiología. El tiempo de hiperlipidemia tampoco estaba establecido, ya que la mayor parte de los pacientes conoció sus niveles de lípidos después del evento coronario.

La efectividad del régimen en el tratamiento de las hiperipidemias es motivo de controversia, existiendo autores que estiman que una dieta baja en colesterol y grasas saturadas lleva a disminuciones promedio de colesterol sanguíneo de 10\%, con un rango de 0 a $20 \% 16,17$. Adicionalmente, el grupo que más se beneficiańa a largo plazo seńa el de los pacientes que ya presentan enfermedad coronaria (prevención secundaria), por una mayor adherencia al cambio de hábitos ${ }^{18}$. Sin embargo, existen opiniones que estiman que tratamientos dietéticos adecuadamente controlados por nutricionistas, no llevan a una reducción del colesterol que vaya más allá de $2 \%$, con una regresión a los valores basales al discontinuar la monitorización, con lo que sólo se pospondná la aparición de cardiopatía coronaria en algunos meses. A ello se agrega el que, estudios de seguimiento de 6 a 15 años no habrían mostrado efecto favorable en el curso de la cardiopatía coronaria sólo con régimen ${ }^{8}$.
La reducción de CLDL de 11,1\% observada en nuestros pacientes, es concordante con los datos reportados por Ramsay ${ }^{19}$, que demostró que dietas intensivas pueden reducir el colesterol sérico en aproximadamente $13 \%$ en pacientes de alto riesgo. En nuestro estudio, esta reducción fue superior a la lograda con una información médica básica, lo que justificaría el enviar a estos pacientes a un programa nutricional. Sin embargo, esta maniobra fue insuficiente, ya que sólo $10 \%$ de ellos logra, al año, los niveles recomendados de CLDL, para los portadores de enfermedad coronaria. Igualmente, no nos es factible obtener conclusiones respecto a la adherencia, ya que nuestros datos son sólo con un seguimiento de un año. Sin embargo, el hecho de que dicha adherencia al cabo de este período sea de sólo $64 \%$ de los pacientes, plantea dudas sobre lo anterior.

Varios estudios han mostrado que regímenes recomendados para reducir el CLDL pueden reducir también el CHDL, hecho que no se produjo en nuestra serie.

Un motivo de preocupación en nuestros pacientes es la falta de modificación del peso y del índice de masa corporal al cabo de un año, lo que hace plantear una respuesta sólo parcial a las indicaciones dietéticas.

La maniobra nutricional utilizada, fue adaptada de la entregada por el «National Cholesterol Education Program», el cual recomienda dos tipos de regímenes (nivel 1 y nivel 2) de acuerdo a las características del perfil lipídico ${ }^{1}$, teniendo como meta cifras de colesterol CLDL para diferentes niveles de riesgo.

Recientemente un estudio de Aquilani ${ }^{20}$, comparando diferentes estrategias terapéuticas, mostró las ventajas de una dieta baja en grasa $\leq 20 \%$ del valor calónico total (VCT) por sobre la del NCEP al disminuir los niveles de CLDL y aumentar los de CHDL Sin embargo, al igual que en nuestros pacientes, no se logran las cifras de colesterol recomendadas para prevención secundaria.

Aun cuando la dieta de nuestra población tiene similitudes con las mostradas en otros estudios nacionales (rica en hidratos de carbono refinados, con una variedad limitada de alimentos y preparaciones), muestra un menor aporte de calorías totales ${ }^{21-23}$.

En nuestros pacientes, la dieta no fue asociada a un programa de ejercicio, hecho que ha demos- 
trado beneficios adicionales ${ }^{24,25}$. Sin embargo, consideramos esto impracticable en nuestra realidad.

Este estudio, hecho en pacientes ambulatorios, reproduce en forma más real lo que sucede en la práctica diaria y muestra las metas a las que es factible llegar con el sólo uso de régimen en nuestra realidad hospitalaria.

Los estudios Euroaspire I y II, que midieron la prevalencia de factores de riesgo en pacientes coronarios en Europa, mostraron igualmente una alta proporción de pacientes con factores de riesgo no controlados ${ }^{27,28}$. En los dos períodos estudiados, el mayor impacto se observó en la reducción del porcentaje de pacientes con colesterol total igual o superior a $5 \mathrm{mmol} / \mathrm{L}(200 \mathrm{mg} / \mathrm{dl})$ de $82,6 \%$ a $55,8 \%$ y que la mayor variación terapéutica en dicho período fue el incremento en el uso de estatinas de $26 \%$ a $61 \%{ }^{28}$. Por otro lado, el programa CHAMP, también en pacientes en prevención secundaria, demostró que la imple-

\section{REFERENCIAS}

1. National Cholesterol Education Program. Third Report of the Expert Panel on Detection, Evaluation, and Treatment of High Blood Cholesterol in Adults (Adult Treatment Panel III). JAMA 2001; 285: 2486-97.

2. Scandinavian Simvastatin Survival Study Group. Randomized trial of Cholesterol lowering in 4444 patients with coronary heart disease: the Scandinavian Simvastatin Survival Study (4S). Lancet 1994; 344: 1383-9.

3. Lewis SJ, Sacks FM, Mitchell JS, Eats C, Giasser S, KeLL S ET AL. Effect of Pravastatin on cardiovascular events in women after MI: The Cholesterol and recurrent events (CARE) Study. J Am Coll Cardiol 1998; 32: 140-6.

4. TonkIN. Long-term Intervention with Pravastatin in Ischemic Disease (LPID study). Am J Cardiol 1995; 76: 474-9.

5. Ciark LT. Treating dyslipidemia with statins: the risk-benefit profile. Am Heart J 2003; 145: 387-96.

6. Stockins A, Candia M, Vilaagrán J, Salman J, Meriño G, Silva A Et AL. Persistencia de factores de riesgo coronario en pacientes sometidos a cirugía de revascularización coronaria. Rev Chil Cardiol 1999; 18: 124. mentación de una terapia sistemática con estatinas, permitió elevar el porcentaje de pacientes con cifras adecuadas de CLDL de $6 \%$ a $58 \% 29$.

En resumen, podemos concluir que: la reducción de niveles de CLDL con una maniobra nutricional estandarizada, es similar a la descrita en otras comunicaciones y mayor a la obtenida tras una información entregada por el médico en su consulta. Al igual que en otros estudios, un porcentaje muy bajo de pacientes logra las metas de CLDL menores a $100 \mathrm{mg} / \mathrm{dl}$, recomendadas por el NCEP. La adherencia al régimen al cabo de un año de tratamiento es sólo regular, no siendo posible entregar información sobre ella a un plazo mayor, dado al tiempo de seguimiento de este estudio. Lo anterior plantea como absolutamente necesario el uso de fármacos hipolipemiantes, como complemento al régimen, en pacientes con enfermedad coronaria en los hospitales públicos chilenos.

7. Yáñez J, Storme O, Stockins B, Lanas F. Persistencia de factores de riesgo coronario en pacientes que han presentado un infarto agudo al miocardio. Rev Chil Cardiol 2001; 20: 275.

8. Воот CP. A cholesterol reducing diet is not useful. Ned Tijdschr Geneeskd 1997; 141: 2539-42.

9. DeNKE MA. Cholesterol-lowering diets. A review of the evidence. Arch Intern Med 1995; 155: 17-26.

10 Schaefer E, Lamon-Fava S, Ausman LM, Ordovas JM, Cievidence BA, Judd JT ET AL. Individual variability in lipoprotein response to National Cholesterol Education Program Step II diet. Am J Clin Nutr 1997; 65: 823-30.

11. Datos adaptados de la Sociedad de Actuarios de U.S.A. (Metropolitan Life Insurance Company, 1959).

12. Keys A, Menotti A, Karvonen MJ, Aravanis C, Biackburn H, Buzina R et al. The diet and 15-year death rate in the Seven Countries Study. Am J Epidemiol 1986; 124: 903-15.

13. Kahn HA, Phiшps RL, Snowdon DA, Choi W. Association between reported diet and all-cause mortality. Twenty-one-year follow-up on 27,530 adult Seventh-Day Adventists. Am J Epidemiol 1984; 119: 775-87.

14. Johansson L, Drevon CA, AA Bjorneboe GE. The Norwegian diet during the last hundred years in 
relation to coronary heart disease. Eur J Clin Nutr 1996; 50: 277-83.

15. Heart Protection Study Collaborative Group. MRC/BHF Heart Protection Study of cholesterol lowering with simvastatin in 20.536 high-risk individuals: a randomized placebo-controlled trial. Lancet 2002; 360: 7-22.

16. Stalenhoef AF. Cholesterol reducing food certainly is useful. Ned Tijdschr Geneeskd 1997; 141: 25435.

17. Puska P, Salonen Jt, Nissinen A, Tounilethto J, VARTIAUNen E, KorHonen H ET aL. change in risk factors for coronary heart disease during 10 years of a community intervention program (North Karelia project). BMJ 1983; 287: 1840-4.

18. Watts GF, Lewis B, Brunt JN, Lewis ES, Coltart DJ, SMITH LD ET AL. Effects on coronary artery disease of lipid-lowering diet, or diet plus cholestyramine, in the St. Thomas' Atheroesclerosis Regression Study (STARS). Lancet 1992; 339: 563-9.

19. RAMSAY LE, Yeo WW, Jackson PR. Dietary reduction of serum cholesterol concentration: time to think again. BMJ 1991; 303: 953-7.

20. Aquilani R, Tramarin R, Pedretti RFE, Berttolotti G, Sommaruga M, Mariani P et al. Despite Good Compliance, very low fat diet alone does not achieve recommended cholesterol goals in outpatients with coronary heart disease. Eur Heart J 1999; 20: 1020-9.

21. Atalah E, Arteaga C, Reboledo A. Consumo de alimentos portadores de antioxidantes naturales en adultos. Rev Chil Nutr 1995; 23: 34-41.

22. Castilo C, Atalah E, Benavides X, Arteaga C. Patrones alimentarios en adultos que asisten a consultorios de atención primaria en la Región Metropolitana. Rev Méd Chile 1997; 125: 283-9.

23. Albala C, Viliarroel P, Olivares S, Trufelo I, Vio F, AndRade M et al. Mujeres obesas de alto y bajo nivel socioeconómico: composición de la dieta $\mathrm{y}$ niveles séricos de lipoproteínas. Rev Méd Chile 1989; 117: 3-9.

24. Nolte LJ, Nowson CA, Dyke AC. Effect of dietary fat reduction and increased aerobic exercise on cardiovascular risk factors. Clin Exp Pharmacol Physiol 1997; 24: 901-3.

25. Rutledge JC, Hyson DA, Garduno D, Cort DA, Paumer L, Kappagoda CT. Lifestyle modification program in management of patients with coronary artery disease: the clinical experience in a tertiary care hospital. J Cardiopulm Rehabil 1999; 19: 226-34.

26. EuROASPIRE. A European Society of Cardiology survey of secondary prevention of coronary heart disease: principal results. Euroaspire Study Group. European Action on Secondary Prevention through Intervention to Reduce Events. Eur Heart J 1997; 18: 1569-82.

27. EUROASPIRE I AND II GROUP. European Action on Secondary Prevention to Reduce Events. Clinical reality of coronary prevention guidelines: a comparison of Euroaspire I and II in nine countries. Euroaspire I and II Group. Lancet 2001; 357: 9951001.

28. Fonarow GC, Gawlinski A, Moughrabi S, Tiшsch $\mathrm{JH}$. Improved treatment of coronary heart disease by implementation of Cardiac Hospitalization Atherosclerosis Management Program (CHAMP). Am J Cardiol 2001; 87: 819-22. 